\title{
Prediction of microstructure composition in steel plate heated using high power Yb:YAG laser radiation
}

\author{
Marcin Kubiak ${ }^{1, *}$ \\ ${ }^{1}$ Czestochowa University of Technology, Institute of Mechanics and Machine design Foundations, \\ Dabrowskiego 73, 42-200 Czestochowa, Poland
}

\begin{abstract}
This work concerns numerical modelling and computer simulations of temperature field and phase transformations during Yb:YAG laser heating of sheets made of S355 steel. The distribution of laser power emitted by Trumpf laser head D70 is used in the analysis. The heat source is modelled on the basis of interpolation algorithms using geostatistical kriging method. Coupled heat transfer and fluid flow in the fusion zone are described respectively by transient heat transfer equation with convective term and Navier-Stokes equation. The kinetics of phase transformations and volumetric fractions of arising phases are obtained on the basis of Johnson-Mehl-Avrami (JMA) and Koistinen-Marburger (KM) models. Continuous Heating Transformation (CHT) diagram is used for heating process and Continuous Cooling Transformation (CCT) diagram is used for heated steel with the decomposition of final volume fractions of phases transformed form austenite dependant on cooling rates.
\end{abstract}

Keywords: laser heating, microstructure composition, heat source, numerical modelling

\section{Introduction}

Laser beam processing of steel has increasing application in many industries, mainly due to high process speed and good quality of the product as well as a small zone of thermal influence on the material. The concentration of a laser heat energy leads to the generation of high cooling rates in heat affected zone (HAZ). As a result phase transformations in solid state are present, which leads to the formation of various structures in HAZ with differing mechanical properties [1,2].

The material in this process is heated to various temperatures. Different structures occur in the heat affected zone (HAZ) as a result of different heating and cooling conditions. Changes in the microstructure covering phase transformations in solid state are the cause of significant changes in the mechanical and operational properties of this zone in comparison to base material [3].

\footnotetext{
*Corresponding author: kubiak@imipkm.pcz.pl

Reviewers: Leszek Radziszewski, Alžbeta Sapietová
} 
Physical phenomena accompanying laser-material interaction is a very complex issue and requires a new approach to the theory and numerical solution techniques. Heat distribution in laser processing proceeds in different conditions in comparison to classic heating techniques. Very important in terms of formal modelling is a proper selection of heat source shape and its energy distribution, especially when the heat source is a solid state laser with the distribution different than classical Gauss distribution [4]. Material flow in the fusion zone affects calculated temperature distribution in heated element $[5,6]$, in consequence having a noticeable influence on estimated microstructure composition. The analysis of the kinetics of phase transformation is carried out by well known mathematical models for diffusive and non-diffusive transformations and Continuous-Cooling Transformations (CCT) diagrams. High heating rates and various maximum heating temperatures of steel occur in the case of laser heating [7-10] affect the kinetics of phase transformation in the solid state. Therefore, the influence of heating and cooling rates, and the impact of maximum temperatures of the thermal cycles on the kinetics of phase transformations should be taken into account in the analysis.

A comprehensive theoretical model of thermal phenomena and phase transformations in solid state during $\mathrm{Yb}$ :YAG heating of S355 steel sheets is presented in this study. Examples of computer simulations include temperature field and estimated structure composition of elements made of S355 steel, heated by Yb:YAG laser. Schematic sketch of analyses system is presented in Fig. 1.

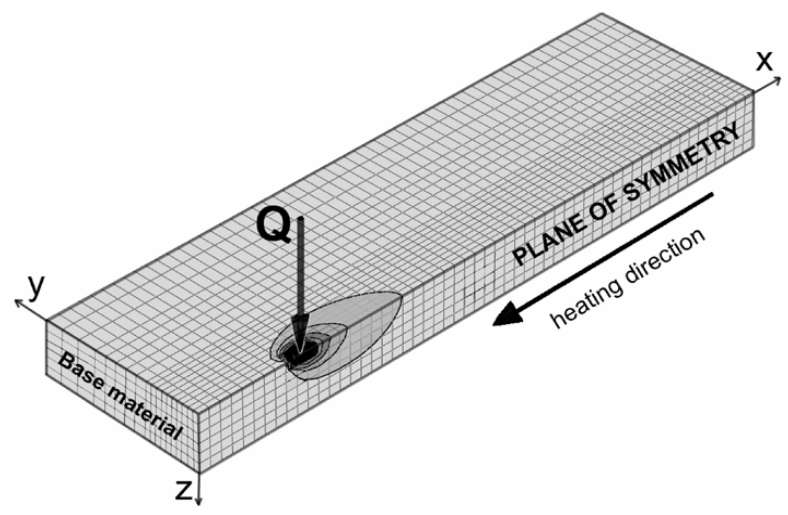

Fig. 1. Sketch of considered system

\section{Mathematical model}

\subsection{Thermal phenomena}

The temperature field is obtained by the solution of transient heat transfer equation with convective term, expressed as follows [11]:

$$
\frac{\partial}{\partial x_{i}}\left(\lambda \frac{\partial T}{\partial x_{i}}\right)=C_{e f}\left(\frac{\partial T}{\partial t}+v_{i} \frac{\partial T}{\partial x_{i}}\right)-\widetilde{Q}
$$

where $T=T\left(x_{i}, t\right)$ is temperature at a point $x_{i}, v_{i}$ is a velocity vector, $\lambda=\lambda(T)$ is a thermal conductivity, $C_{e f}=C_{e f}(T)$ is an effective heat capacity with latent heat of fusion and evaporation taken into account, $\widetilde{Q}$ is a volumetric heat source with laser beam and electric arc heat sources power distributions taken into considerations. 
Initial condition: $t=0: T=T_{0}$ and boundary conditions complete equation (1), taking into account heat loss due to convection, radiation and evaporation

Velocity field of liquid material in the melted zone is obtained by the solution of Navier-Stokes equation fulfilling the continuity equation with buoyancy forces and flow through porous medium taken into consideration

$$
\begin{gathered}
\frac{\partial \rho}{\partial t}+\frac{\partial}{\partial x_{i}}\left(\rho v_{i}\right)=0 \\
\frac{\partial\left(\rho v_{i}\right)}{\partial \mathrm{t}}+\frac{\partial}{\partial x_{j}}\left(\rho v_{i} v_{j}\right)=-\frac{\partial p}{\partial x_{i}}+\frac{\partial}{\partial x_{j}}\left(\mu \frac{\partial v_{i}}{\partial x_{j}}\right)+\mathbf{g} \beta_{T}\left(T-T_{r e f}\right)-\frac{\mu}{\rho K} v_{i}
\end{gathered}
$$

where: $\rho$ is a density, $\mathbf{g}$ is acceleration of gravity, $\beta_{T}$ is a volume expansion coefficient due to heating, $T_{\text {ref }}$ is a reference temperature, $\mu$ is a dynamic viscosity, $K$ is porous medium permeability.

Equation (3) is completed by initial condition $t=0: \mathbf{v}=0$ and boundary conditions implemented at the welding pool boundary determined by solidus temperature $\left(T_{r e f}=T_{S}\right)$ [5].

Interpolation algorithms are used for the description of $\mathrm{Yb}: \mathrm{YAG}$ laser beam heat source power distribution. Ordinary Kriging method [3,12] is used in the numerical algorithm, taking into account the real laser power distribution obtained in experimental research made on laser welding station equipped with TruDisk 12002 laser.

\subsection{Phase transformation in solid state}

A volumetric fraction of forming austenite during heating is described by Johnson-MehlAvrami (JMA) model, expressed as follows:

$$
\tilde{\eta}_{A}(T, t)=\eta_{(\cdot)}\left(1-\exp \left(-b t^{n}\right),\right.
$$

where $\eta_{(.)}$is a sum of volumetric fractions of base material phase composition $\left(\eta_{(.)}=1\right)$, coefficients $b=b(T)$ and $n=n(T)$ are determined by start $\left(\eta_{s}=0.01\right)$ and final $\left(\eta_{f}=0.99\right)$ conditions for phase transformations:

$$
b(T)=-\frac{\ln \left(\eta_{f}\right)}{\left(t_{s}\right)^{n(T)}}, \quad n(T)=\frac{\ln \left(\ln \left(\eta_{f}\right) / \ln \left(\eta_{s}\right)\right)}{\ln \left(t_{s} / t_{f}\right)},
$$

where $t$ is a time, $t_{s}=t_{s}\left(T_{s A}\right)$ and $t_{f}=t_{f}\left(T_{f A}\right)$ are start and finish times of a phase transformation, $T_{S A}$ and $T_{f A}$ are corresponding start and finish temperatures.

Phase fractions forming during the cooling process are determined by the temperature and cooling rate $\left(v_{8 / 5}=\left(800^{\circ} C-500^{\circ} C\right) / t_{8 / 5}\right)$ in the temperature range [800-500] ${ }^{\circ} \mathrm{C}$, where $t_{8 / 5}$ is a cooling time. A fraction of the new phase like: ferrite, pearlite and bainite (diffusive transformations) are determined on the basis of JMA formula taking into account already existing phase fractions:

$$
\eta_{(\cdot)}(T, t)=\eta_{(\cdot)}^{\%} \widetilde{\eta}_{A}\left(1-\exp \left(-b(t(T))^{n}\right)\right), \underline{\eta}_{A}-\sum_{k} \eta_{k} \geq 0, \sum_{k} \eta_{k}^{\%}=1
$$


where: $\eta_{(\cdot)}^{\%}$ is the maximum fraction for determined cooling rate, estimated on the basis of microstructure analysis of examined steel, $\eta_{A}$ is austenite fraction due to heating process, $\eta_{k}$ is a phase fraction formed earlier in the cooling process, coefficients $b$ and $n$ are also determined using formula (2), in which $b=b\left(T\left(\mathrm{v}_{8 / 5}\right)\right)$ and $n=n\left(T\left(\mathrm{v}_{8 / 5}\right)\right)$.

Volumetric fraction of martensite $(\eta M)$ below $M_{s}$ temperature is estimated according to the following Koistinen-Marburger (KM) equation:

$$
\eta_{M}(T)=\eta_{(\cdot)}^{\%}\left(1-\exp \left(-k\left(M_{s}-T\right)\right)\right), T \in\left[M_{s}, M_{f}\right]
$$

Coefficient $k$ depends on martensite phase start and final temperatures ( $M_{s}$ and $M_{f}$ ) determined also basing on CCT diagram:

$$
k=-\frac{\ln \left(\eta_{s}\right)}{M_{s}-M_{f}}=-\frac{\ln (0.01)}{M_{s}-M_{f}}
$$

The heating rate significantly affects the temperature of austenite formation. Austenitization temperatures $A c_{1}$ and $A c_{3}$ significantly increase As a result of high heating rates up to the maximum temperature in the process (reaching several thousands $\mathrm{K} / \mathrm{s}$ in laser processing) [13].

Differential equations describing thermal phenomena in discussed welding processes are numerically solved using projection method with finite volume method (FVM) [14]. The spatial variables are discretized using staggered grid to avoid odd-even decoupling between the pressure and velocity. Numerical algorithms are implemented into computer solver using ObjectPascal programming language.

\section{Results}

Simulations of the Yb:YAG laser heating process are performed for S355 steel sheets with dimensions $250 \mathrm{~mm}$ in length, $50 \mathrm{~mm}$ in width, with a thickness of $5 \mathrm{~mm}$, assuming laser head movement speed $v=3 \mathrm{~m} / \mathrm{min}$, the focusing point on the top surface of heated element $(z=0)$ and laser power: $Q=900 \mathrm{~W}$. The analysed domain is discretized by a staggered grid with the spatial step set to $0.1 \mathrm{~mm}$.

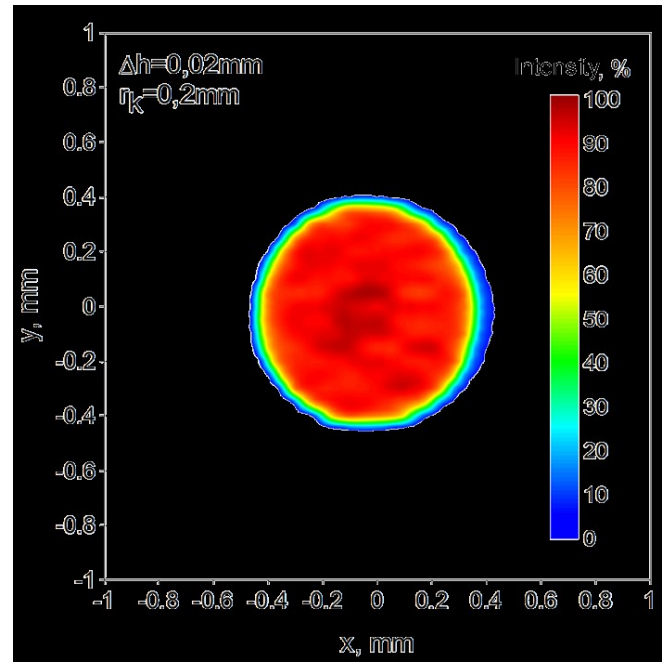

Fig. 2. Percentage distribution of Yb:YAG laser beam power 


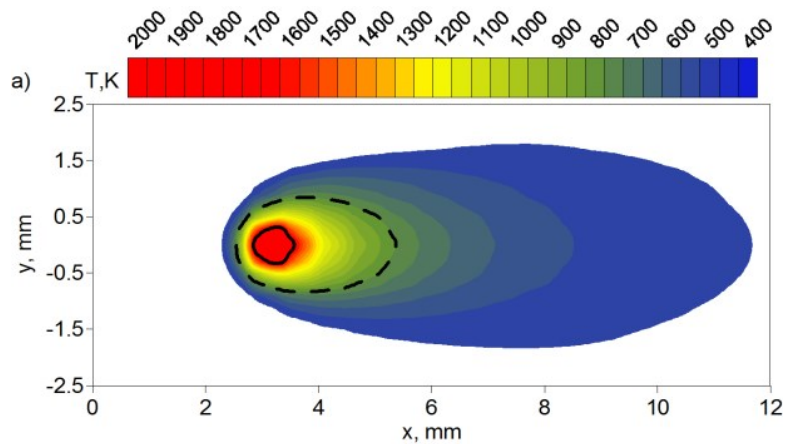

b)

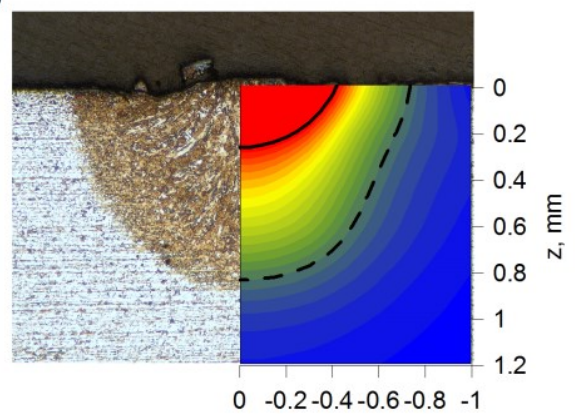

$\mathrm{y}, \mathrm{mm}$

Fig. 3. Temperature distribution a) at the top surface and b) in cross section (for $x=3 \mathrm{~mm}$ ) of laser heated sheet in comparison with macroscopic picture of the real heated element
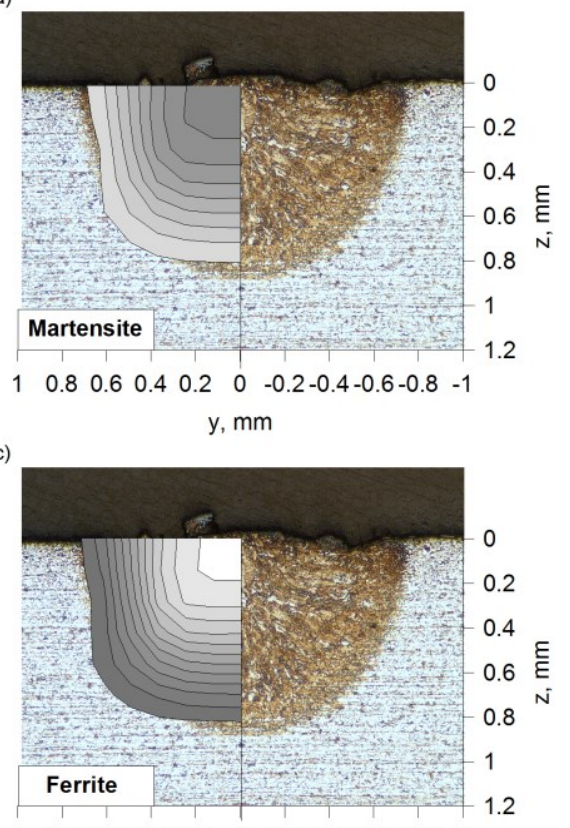

$\begin{array}{lllllllllll} & 0.8 & 0.6 & 0.4 & 0.2 & 0 & -0.2 & -0.4-0.6 & -0.8 & -1\end{array}$

$$
\mathrm{y}, \mathrm{mm}
$$

b)
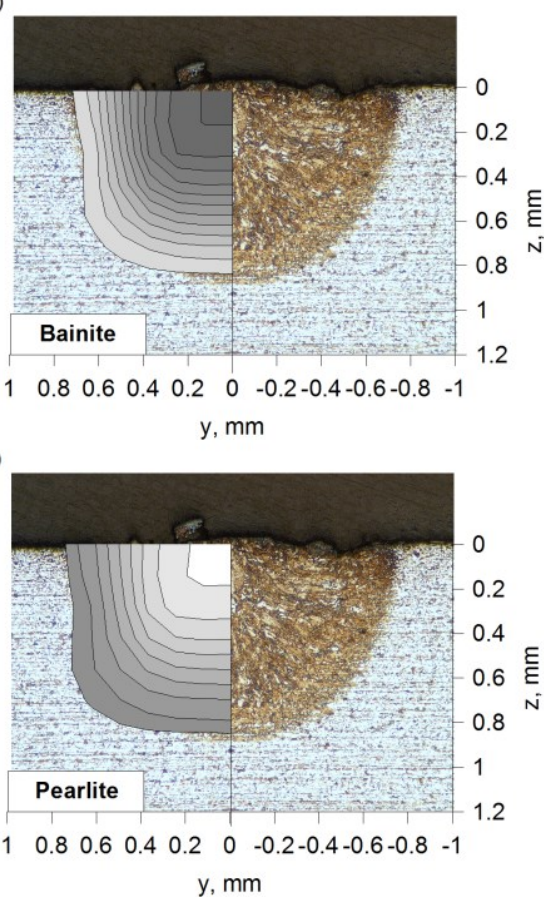

fraction

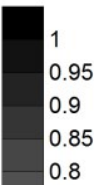

0.75

0.7

0.65

0.6

0.55

0.5

0.45

0.4

0.35

0.3

0.25

0.2

0.15

0.1

0.05

Fig. 4. Volumetric fractions of structure constituents: a) martensite, b) bainite, c) ferrite and d) pearlite 


\section{Conclusions}

Heat affected zone in laser heated elements is narrow, up to about $0.8 \mathrm{~mm}$ in radius at the top surface of heated element. It can be observed that heated element is melted in the area of $0.4 \mathrm{~mm}$ in radius with about $0.2 \mathrm{~mm}$ in the direction of heat source penetration into the material. The use of lower laser beam generated by Yb:YAG laser radiation contributed to the reduction of melted area and heat affected zone.

In the thermal influence zone the steel is composed mostly of bainite and martensite $(60 \% / 40 \%)$. The material is partially hardened, having maximum martesite fraction about $80 \%$ in the heat source activity zone.

Application of Kriging algorithm allowed to use experimental studies in numerical analysis and for a precise reproduction of the real thermal load. Obtained structure constituents in the cross section of heated element well agree with experimentally obtained macroscopic picture.

\section{References}

1. Y.C. Kim, M. Hirohata, K. Inose, Welding in the World, no 3, 64 (2012)

2. J. Pilarczyk, Welding metallurgy, Wyd. Pol. Warszawskiej, Warszawa (1977)

3. W. Piekarska, Archives of Metallurgy and Materials, 4, 60 (2015)

4. M. Kubiak, W. Piekarska, S. Stano, International Journal of Heat and Mass Transfer, 83 (2015)

5. J. Zhou, H.L. Tsai, International Journal of Heat and Mass Transfer, 51 (2008)

6. A. De, T. Debroy, Welding Journal, 84 (2005)

7. O. Grong, Metallurgical Modelling of Welding, The Institute of Materials, Cambridge (1997)

8. S. Serajzadeh, Journal of Materials Processing Technology, 146 (2004)

9. W. Zhang, B. Wood, T. DebRoy, et al., Acta Materialia, 51, (2003)

10. T. Domański, A. Bokota, International Journal of Mechanical Sciences 96-97, 47 (2015)

11. M. Kubiak, W. Piekarska, Z. Saternus, T. Domański, Procedia Engineering, 136 (2016)

12. M.A. Oliver, R. Webster, International Journal of Geogr Inf Sys, 4 (1990)

13. M Kubiak, W Piekarska, Computers \& Structures, 172 (2016)

14. S.V. Patankar, Numerical heat transfer and fluid flow, Taylor \& Francis, USA (1990) 